\title{
Salvage pulmonary resection after stereotactic body radiotherapy: A feasible and safe option for local failure in selected patients
}

\author{
Mara B. Antonoff, MD, ${ }^{a}$ Arlene M. Correa, PhD, ${ }^{a}$ Boris Sepesi, MD, ${ }^{a}$ Quynh-Nhu Nguyen, MD, ${ }^{b}$ \\ Garrett L. Walsh, MD, ${ }^{a}$ Stephen G. Swisher, MD, ${ }^{a}$ Ara A. Vaporciyan, MD, ${ }^{a}$ Reza J. Mehran, MD, ${ }^{a}$ \\ Wayne L. Hofstetter, MD, ${ }^{a}$ and David C. Rice, $\mathrm{MB}, \mathrm{BCh}^{\mathrm{a}}$
}

\begin{abstract}
Objective: For inoperable patients with pulmonary malignancy, stereotactic body radiotherapy is a reasonable therapeutic option. Despite good early tumor control, local failure occurs in up to $10 \%$ of patients by 3 years. Because management of local recurrence after stereotactic body radiotherapy is unclear, we evaluated use of surgery as a salvage option.
\end{abstract}

Methods: A retrospective review was conducted of consecutive patients from a single institution who underwent salvage resection of primary and metastatic pulmonary malignancies previously treated with stereotactic body radiotherapy. In addition, a literature search was conducted to identify previous reports of pulmonary resection for local stereotactic body radiotherapy failures, to allow cumulative analyses with previously published cases.

Results: A total of 21 patients met inclusion criteria. The median time between stereotactic body radiotherapy and salvage surgery was 16.2 months (range, 6.4-71.5). Postoperative complications occurred in 7 patients (18.9\%), in whom atrial arrhythmias and prolonged air leaks ( $>5$ days) were most frequent $(\mathrm{n}=2$ each, $5.4 \%$ ). There was no local recurrence after salvage surgery. Distant failure occurred in 5 of 21 patients $(23.8 \%)$ at a median of 36.2 months, and median disease-free survival was 19.2 months. The 30- and 90-day mortality was $4.8 \%$ (1 patient). Cumulative analysis included 37 patients from 4 institutions and comprised $26(78.8 \%)$ primary non-small cell lung cancers and 11 $(29.7 \%)$ lung metastases. Median overall survival after salvage surgery was 46.9 months, and 3-year survival was $71.8 \%$.

Conclusions: After local failure of stereotactic body radiotherapy, salvage resection remains a viable option for operable patients, with acceptable morbidity and survival. As use of stereotactic body radiotherapy continues to expand, further studies to evaluate the optimal management for local failure are needed. (J Thorac Cardiovasc Surg 2017;154:689-99)

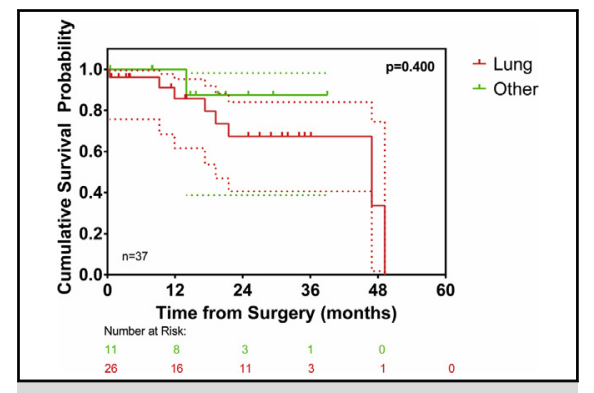

Survival after salvage surgery for failed SBRT.

\section{Central Message}

Operative resection after local failure of SBRT in highly select individuals is feasible and safe, and has an overall acceptable morbidity and mortality.

\section{Perspective}

This study represents the largest series of pulmonary resection after local SBRT failure reported to date, along with a cumulative review that incorporates all patients who have been previously reported. We demonstrate that resection after local failure of SBRT in highly select individuals is feasible and safe, and has an overall acceptable morbidity and mortality, albeit higher than what is typically observed in nonirradiated patients.

See Editorial Commentary page 700 .
For early-stage non-small cell lung cancer (NSCLC), operative resection consisting of lobectomy and mediastinal lymph node dissection has been a long-standing, established standard of care. For patients thought to be

\footnotetext{
From the Departments of ${ }^{\mathrm{a}}$ Thoracic and Cardiovascular Surgery and ${ }^{\mathrm{b}}$ Thoracic Radiation Oncology, University of Texas MD Anderson Cancer Center, Houston, Tex. Read at the 96th Annual Meeting of The American Association for Thoracic Surgery, May 14-18, 2016, Baltimore, Maryland.

Received for publication May 2, 2016; revisions received Feb 23, 2017; accepted for publication March 25, 2017; available ahead of print May 9, 2017.

Address for reprints: Mara B. Antonoff, MD, 1400 Pressler St, Unit 1489, Houston,

TX 77030 (E-mail: mbantonoff@mdanderson.org).

$0022-5223 / \$ 36.00$

Copyright (c) 2017 by The American Association for Thoracic Surgery

http://dx.doi.org/10.1016/j.jtcvs.2017.03.142
}

inoperable or with prohibitively high operative risk, novel therapeutic options have been introduced in recent years through advances in radiotherapy. ${ }^{2}$ Stereotactic body radiotherapy (SBRT) is a means of delivering high doses of external beam radiation over a limited number of treatment fractions to an image-defined target, and it has become a

Scanning this QR code will take you to a supplemental video. To view the AATS 2016 Webcast, see the URL next to the webcast thumbnail. 


\section{Abbreviations and Acronyms \\ $\mathrm{CT}=$ computed tomography \\ $\mathrm{DLCO}=$ diffusing capacity of the lung for carbon monoxide \\ FEV1 = forced expiratory volume in 1 second \\ FDG = fluorodeoxyglucose \\ GT $=$ General Thoracic \\ MDACC $=$ MD Anderson Cancer Center \\ NSCLC $=$ non-small cell lung cancer \\ PET = positron emission tomography \\ SBRT = stereotactic body radiotherapy \\ STS = Society of Thoracic Surgeons}

viable option for the management of patients with early NSCLC unable to tolerate surgical resection. ${ }^{2-4}$ Several single-institution studies have demonstrated SBRT to be an efficacious and well-tolerated treatment strategy in medically inoperable patients with early-stage lung cancer. $^{3,5,6}$ The Radiation Therapy Oncology Group 0236 trial, subsequently published in 2010, prospectively evaluated SBRT among patients at multiple North American centers, revealing high rates of local tumor control with acceptable treatment-related morbidity. ${ }^{7}$ Other investigators have further corroborated these findings, with reproducibly acceptable rates of intermediate-term local control and minimal toxicities. . $^{8-10}$

Given the success of using SBRT for stage I NSCLC in medically inoperable patients, increasing interest has arisen regarding the potential application of this modality for healthier, potentially operable patients. ${ }^{11-13}$ Although some early studies have demonstrated encouraging evidence, they have been inconclusive, and despite the initiation of several randomized trials, accrual has been a challenge. ${ }^{11}$ In addition, the use of SBRT has been extended in a number of centers to include pulmonary metastases, in addition to primary lung cancer. Important issues warranting investigation will include determination of the ideal means of following patients radiographically after SBRT and establishing protocols for intervention on local failure.

The outcomes for salvage lung resection after SBRT are not well established. Previous experiences have been described in 4 small case series, with a suggestion of reasonable perioperative morbidity and local control, among patients with NSCLC and those undergoing treatment for pulmonary metastatic disease. ${ }^{14-17}$

In this study, we aimed to evaluate our experience with operative lung resection in patients in whom SBRT has failed. Further, we review the combined outcomes in patients from our center along with those previously reported in the published literature.

\section{MATERIALS AND METHODS}

\section{Anderson Cancer Center Institutional Analysis}

After obtaining Institutional Review Board approval (PA-0169), we used our departmental surgical database to identify all patients who underwent salvage pulmonary resection after prior radiation between January 1 , 2009, and September 30, 2015. Patient medical records were reviewed, and only patients in whom the lesion in question was previously treated with SBRT and then underwent salvage resection for local recurrence were selected. Patients were excluded if the prior radiation was inconsistent with SBRT techniques or if the SBRT was delivered to a lesion other than that which was subsequently resected. All patients underwent surgery at MD Anderson Cancer Center (MDACC); however, not all patients received SBRT at our institution. Among 716 patients who underwent pulmonary resection after previous radiotherapy, only 21 were identified who met inclusion criteria, and these patients comprised the MDACC study cohort.

Detailed patient data were retrospectively collected from our departmentally maintained, prospectively entered database and supplemented with additional chart review. Variables collected included information pertaining to demographics, histology, staging, radiation dosing and fractions, patient comorbidities and operability, extent of resection, recurrence, and vital status.

\section{Aggregate Analysis}

In addition, a MEDLINE search was performed to identify all previous reports of surgical resection after local failure after initial treatment with SBRT. Searches were performed in September 2015, limited to English language literature, and using the following search terms: "salvage surgery," "salvage resection," along with "lung" and "pulmonary," as well as "SBRT," "SABR," and "stereotactic radiation." This resulted in 56 articles, which were reviewed by 2 of the authors (MBA and DCR) to specifically identify publications regarding lung resection after local recurrence after SBRT. Four studies were identified, totaling 18 patients; however, because Taira and colleagues ${ }^{17}$ included resection of specimens lacking any residual disease, we chose to use only the other 3 reports for cumulative analyses. ${ }^{14-17}$ From these previous publications, data were extracted for each patient, including age, sex, histology, staging, operability, radiation dosing and fractions, extent of resection, any other available operative details, and disease status/vital status at last follow-up. The data from the patients in these publications were combined with those of the MDACC cohort, thus constituting the final aggregate cumulative group.

For analyses of both the MDACC and the cumulative groups, continuous variables are reported as median and range, and categoric variables are presented as $\mathrm{N}$ and $\%$. Student paired $t$ tests were used to compare pre-SBRT and presalvage surgery pulmonary function test results, with a 2 -sided $P$ value of .05 considered significant. Kaplan-Meier analyses were performed to evaluate survival from the time of surgery and survival from completion of SBRT. All statistical analyses were performed using IBM SPSS statistics version 23.0 (IBM Corporation, Armonk, NY).

\section{RESULTS Patients}

At MDACC, 21 individuals met inclusion criteria and comprise the MDACC group, consisting of 15 patients $(71.4 \%)$ with primary NSCLC and 6 patients $(28.6 \%)$ with pulmonary metastatic disease (Table 1). The cumulative group included 37 patients at 4 institutions, including $26(70.2 \%)$ with NSCLC (17 [65.4\%] squamous cell, 7 [26.9\%] adenocarcinoma, and 2 [7.7\%] NSCLC not otherwise specified). Eleven patients (29.7\%) underwent pulmonary resection for recurrent metastases, including 7 
TABLE 1. Patients: Summary of MD Anderson Cancer Center and cumulative groups

\begin{tabular}{lcc}
\hline & MDACC group & Cumulative group $^{12-14}$ \\
\hline Total patients & 21 & 37 \\
NSCLC & $15(71.4 \%)$ & $26(70.3 \%)$ \\
Squamous cell & $10(66.7 \%)$ & $17(65.4 \%)$ \\
Adenocarcinoma & $4(26.7 \%)$ & $7(26.9 \%)$ \\
NSCLC-NOS & $1(6.7 \%)$ & $2(7.7 \%)$ \\
Metastatic disease & $6(28.6 \%)$ & $11(29.7 \%)$ \\
Colorectal & $4(66.7 \%)$ & $7(63.6 \%)$ \\
Esophageal & $1(16.7 \%)$ & $2(18.2 \%)$ \\
Thyroid & $1(16.7 \%)$ & $1(9.1 \%)$ \\
Pharyngeal & & $1(9.1 \%)$ \\
Men & $11(52.4 \%)$ & $21 / 31 \%(67.7 \%)$ \\
Age, y & $73(45-89)$ & $73(45-85)$ \\
Medically inoperable & $4(19.0 \%)$ & $9(24.3 \%)$ \\
\hline
\end{tabular}

MDACC, MD Anderson Cancer Center; NSCLC, non-small cell lung cancer; NOS, not otherwise specified. *Sex unavailable for 4 patients.

$(63.6 \%)$ colorectal, $2(18.2 \%)$ esophageal, 1 thyroid $(9.1 \%)$, and $1(9.1 \%)$ pharyngeal metastases. Tumor measurements were available for 32 patients before SBRT, with a median size of $16.5 \mathrm{~mm}$ (range, 7-40 mm). Data from pretreatment positron emission tomography (PET) were available for 28 individuals whose tumors displayed a maximum fluorodeoxyglucose (FDG) avidity of 5.4 (range, 1-13.7) maximum standardized uptake value.

The MDACC and cumulative groups included 11 men $(52.4 \%)$ and 21 men $(56.8 \%)$, respectively. The median age among all patients was 72.5 years, ranging from 45 to 89 years. Within the cumulative group, approximately one quarter of patients were considered medically inoperable at the time of initial evaluation. Detailed comorbidities are available for the 21 patients in the MDACC group and are shown in Table 2 . Heavy smoking history was highly prevalent, with 6 patients $(28.6 \%)$ having the diagnosis of chronic obstructive pulmonary disease. Before any treatment, the median forced expiratory volume in 1 second (FEV1) was $67 \%$ of predicted, and the median diffusing capacity of the lung for carbon monoxide (DLCO) was $56 \%$

TABLE 2. Comorbidities, MD Anderson Cancer Center group $(\mathbf{n}=\mathbf{2 1})$

\begin{tabular}{lc}
\hline & $\mathbf{N}(\%)$ \\
\hline Coronary artery disease & $2(9.5)$ \\
Cardiac arrhythmia & $3(14.3)$ \\
Hypertension & $11(52.4)$ \\
Cerebrovascular disease & $1(4.8)$ \\
Diabetes mellitus & $4(19.0)$ \\
COPD & $6(28.6)$ \\
History of smoking & $19(90.5)$ \\
Pack-years & $36.9(5-86)$ \\
Sarcoidosis & $1(4.8)$ \\
Significant weight loss* & $2(9.5)$ \\
\hline COPD, Chronic obstructive pulmonary disease. ${ }^{*}$ Within 3 months preoperatively.
\end{tabular}

TABLE 3. Pulmonary function, MD Anderson Cancer Center group $(\mathbf{n}=\mathbf{2 1})$

\begin{tabular}{lccc}
\hline & Pre-SBRT & Presalvage surgery & $\boldsymbol{P}$ value \\
\hline FEV1, liters & $1.84(0.73-3.37)$ & $1.99(0.62-3.33)$ & .123 \\
FEV1, \% predicted & $73(33-130)$ & $69(29-133)$ & .282 \\
DLCO, \% predicted & $67(42-105)$ & $59(42-84)$ & .028 \\
\hline SBRT, Stereotactic body radiation therapy; $F E V 1$, forced expiratory volume in 1 sec- \\
ond; $D L C O$, diffusing capacity of the lung for carbon monoxide.
\end{tabular}

of predicted (Table 3). Additional common comorbidities were cardiovascular in nature.

The reasons for initial nonoperative therapy are available for the 21 patients in the MDACC cohort. Five patients $(23.8 \%)$ were deemed inoperable, including 1 deemed inoperable at an outside hospital and 4 with predicted postoperative FEV1 or DLCO less than $40 \%$ in all cases. For the 16 patients in the MDACC cohort who were thought to be operable but high risk, these included 10 patients with NSCLC, among whom 4 were offered resection and did not want surgery. Three patients had concomitant additional malignancies requiring aggressive treatment (pancreatic cancer, gastric cancer, and contralateral lung cancer). Of the remaining patients, 1 had a previous lobectomy, borderline spirometry, and was deemed high risk by the cardiologist because of severe coronary artery disease. The other 2 patients included 1 with a previous lobectomy and on chronic anticoagulation and 1 who had been previously treated with chemoradiation for $\mathrm{N} 2$ disease without a known primary, with subsequent discovery of the primary nodule. For the 6 patients in the MDACC cohort with metastatic disease, 2 were evaluated and treated with stereotactic radiation at outside hospitals, and 1 was evaluated at our institution but did not have thoracic surgical consultation before radiation. The other 3 elected to attempt stereotactic radiation on patient choice after having undergone previous thoracotomies (for pulmonary and esophageal resections).

\section{Radiotherapy}

The median radiation doses received in the MDACC group and the cumulative group were both $50 \mathrm{~Gy}$, delivered in 4 fractions (range, 48-70 Gy; 3-15 fractions) (Tables 4 and 5). FEV1 results did not differ significantly after SBRT (Table 3 and Figure 1), although there was a slight diminution of DLCO after radiotherapy, from an average

TABLE 4. Treatment details

\begin{tabular}{lcc}
\hline & MDACC group & Cumulative group $^{12-14}$ \\
\hline Radiation dose & $50(48-70)$ & $50(48-70)$ \\
Fractions & $4(4-15)$ & $4(3-15)$ \\
SBRT to surgery (mo) & $16.2(6.4-71.5)$ & $16.1(6.4-104.0)$ \\
Sublobar resection & $8(38.1 \%)$ & $9(24.3 \%)$ \\
Lobectomy & $12(57.1 \%)$ & $27(73.0 \%)$ \\
Pneumonectomy & $1(4.8 \%)$ & $1(2.7 \%)$ \\
\hline
\end{tabular}

MDACC, MD Anderson Cancer Center; SBRT, stereotactic body radiation therapy. 
TABLE 5. Radiation treatment details

\begin{tabular}{|c|c|c|}
\hline Radiation dose (Gy) & Fractions & BED (Gy) \\
\hline 50 & 4 & 112.5 \\
\hline 50 & 4 & 112.5 \\
\hline 50 & 4 & 112.5 \\
\hline 50 & 4 & 112.5 \\
\hline 50 & 4 & 112.5 \\
\hline 50 & 4 & 112.5 \\
\hline 50 & 4 & 112.5 \\
\hline 50 & 4 & 112.5 \\
\hline 70 & 10 & 119 \\
\hline 50 & 4 & 112.5 \\
\hline 60 & 10 & 96 \\
\hline 50 & 4 & 112.5 \\
\hline 50 & 4 & 112.5 \\
\hline 50 & 4 & 112.5 \\
\hline 50 & 4 & 112.5 \\
\hline 50 & 4 & 112.5 \\
\hline 50 & 4 & 112.5 \\
\hline 48 & 4 & 105.6 \\
\hline 50 & 4 & 112.5 \\
\hline 48 & 4 & 105.6 \\
\hline 52.5 & 15 & 70.875 \\
\hline 48 & 4 & 105.6 \\
\hline 48 & 4 & 105.6 \\
\hline 48 & 4 & 105.6 \\
\hline 48 & 4 & 105.6 \\
\hline 48 & 4 & 105.6 \\
\hline 48 & 4 & 105.6 \\
\hline 48 & 4 & 105.6 \\
\hline 60 & 8 & 105 \\
\hline 48 & 4 & 105.6 \\
\hline 54 & 3 & 151.2 \\
\hline 48 & 4 & 105.6 \\
\hline 48 & 4 & 105.6 \\
\hline 50 & 10 & 75 \\
\hline 48 & 4 & 105.6 \\
\hline 48 & 4 & 105.6 \\
\hline 48 & 4 & 105.6 \\
\hline
\end{tabular}

$B E D$, Biologically effective dose.

of $67 \%$ to $59 \%$ of predicted $(P=.028)$. Median tumor size after SBRT was $35 \mathrm{~mm}(1.5-61 \mathrm{~mm})$. PET data between SBRT and resection were available for 24 individuals, among whom the median maximum standardized uptake value was 9 (1.9-23.5).

\section{Detection of Recurrent Disease}

For the 21 patients in the MDACC cohort, all individuals demonstrated radiologic evidence of recurrence, and 14 patients $(66.7 \%)$ underwent definitive histologic confirmation before operative resection. In these 14 patients, recurrent disease was suspected by increased FDG avidity in 10 patients $(71.4 \%)$ and increased size on computed tomography (CT) scan in 4 patients (28.6\%). Among those patients who did not have histologically proven recurrence preoperatively, all 7 demonstrated increased size on cross-sectional imaging and increased FDG avidity on PETs.

\section{Surgical Resection}

Salvage surgery was performed at a median of 16 months after completion of SBRT, with a range of 6.4 to 104 months (Table 4). Twenty-seven patients $(73.0 \%)$ underwent lobectomy, 9 patients $(24.3 \%)$ underwent sublobar resection (3 wedges, 3 segmentectomies), and 1 patient $(2.7 \%)$ underwent bilobectomy and subsequently required completion pneumonectomy. Three $(8.1 \%)$ operations were performed via minimally invasive approaches (2 thoracoscopic and 1 robotic assisted), as demonstrated in Video 1. Adhesions were noted intraoperatively for nearly all patients $(36 / 37$, $97.3 \%$ ). Degree of adhesions was available for the MDACC group, among whom $10(47.6 \%)$ had adhesions described as extensive or severe. Final pathology resulted in upstaging for 9 of 15 patients (60\%) in the MDACC group (Table 6). All surgical margins were free of malignant disease.

Early postoperative outcomes were available for the 21 patients in the MDACC group (Table 7). Two patients $(9.5 \%)$ required postoperative admission to the intensive care unit and stayed for a median of 5.5 days. The most common complications were pulmonary in nature, occurring in 7 patients $(33.3 \%)$. Two patients $(9.5 \%)$ had a prolonged air leak, which persisted for a median of 14 days. Two patients $(9.5 \%)$ were discharged home on oxygen. Atrial arrhythmias occurred in 3 patients $(14.3 \%)$, whereas other types of complications were less common. One patient $(4.8 \%)$ who underwent bilobectomy with pulmonary arterial patch angioplasty because of calcified nodes adherent to the pulmonary artery (and not thought to be related to previous SBRT) developed acute pulmonary artery thrombosis of the pulmonary artery postoperatively, which required completion pneumonectomy. This patient subsequently developed acute respiratory distress syndrome and died of multiple organ failure.

\section{Recurrence and Survival Outcomes}

For patients who underwent surgical resection at MDACC, surveillance cross-sectional imaging was generally performed with PET or CT scans every 3 months along with a clinic visit, with a mean follow-up time of 17.3 months. During follow-up, the population at risk is composed of 21 patients at MDACC who were followed for 3 years. Distant recurrence developed in 5 patients. The 3-year cumulative incidence of distant recurrence is 5 cases per 21 patients at a risk of $23.8 \%$. All recurrences were distant in nature. The median time to recurrence was 36.2 months, and the median disease-free survival among patients at MDACC was 19.2 months.

Survival curves from the date of SBRT and the date of surgery are shown in Figures 2 and 3. Median survival 

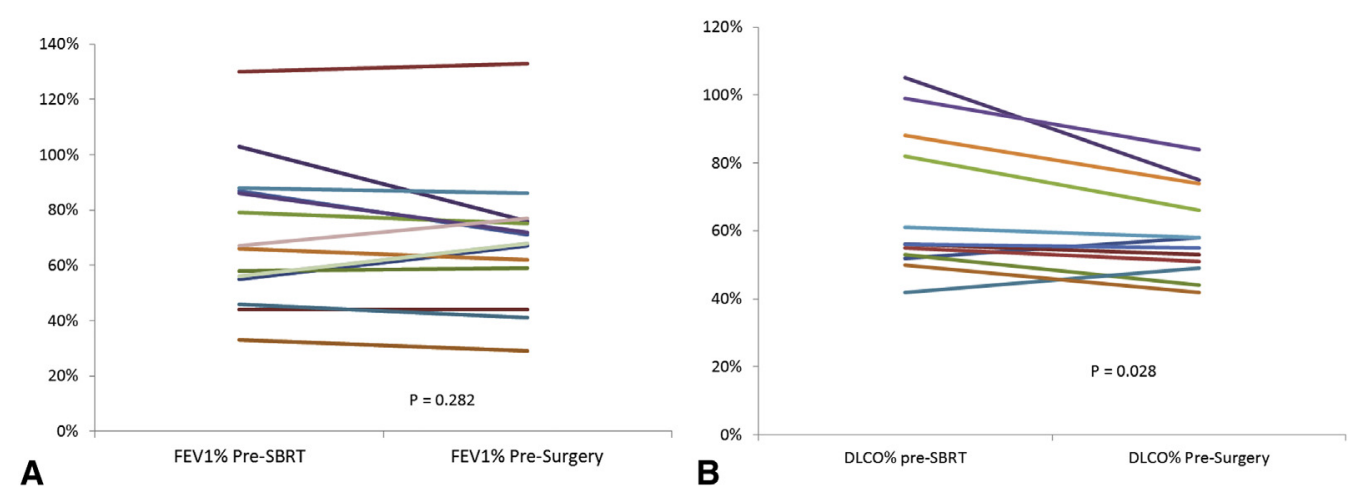

FIGURE 1. Spirometry results for patients at MDACC are shown both before and after receipt of SBRT. A, FEV1 did not change significantly over the course of radiotherapy. B, There was a finding of diminishing DLCO after radiation. FEV1, Forced expiratory volume in 1 second; SBRT, stereotactic body radiation therapy; $D L C O$, diffusing capacity of the lung for carbon monoxide.

from surgery for the MDACC group was 46.9 months, with a 3 -year survival of $53.2 \%$. The 30-day and 90day mortalities were both $1(4.8 \%)$. In the cumulative group, median survival was 46.9 months and 3-year survival was $71.8 \%$.

Subgrouped by primary site of disease, patients in the MDACC group who underwent salvage resection of lung cancer had a median and 3-year survival of 13.6 months and $43.1 \%$, respectively, whereas the corresponding values for the cumulative group were 18.4 months and $67.4 \%$, respectively. For patients in the MDACC group who underwent resection for pulmonary metastatic disease, 3-year survival could not be determined because of limited follow-up time; however, 3-year survival was $87.5 \%$ in the cumulative group. Median survival was not reached during the follow-up period for patients who underwent salvage resection of pulmonary metastatic disease.

\section{DISCUSSION}

Over the last decade, more than 1400 cases of lung SBRT have been performed at MDACC, including 1300 cases of NSCLC and approximately 100 cases of pulmonary metastatic disease. Internal case review has led to the identification of 38 local-only recurrences, which appears to be approximately $2.7 \%$ and amounts to significantly less than most reported rates in the literature. However, the exact rate of local recurrence is unclear, and it has been reported by other authors to be in the range of $10 \%$ to $15 \%$. ${ }^{18,19}$ Part of the difficulty in defining the true rate of recurrence after stereotactic radiation is consequent to the nonuniform radiographic surveillance practices after SBRT, coupled

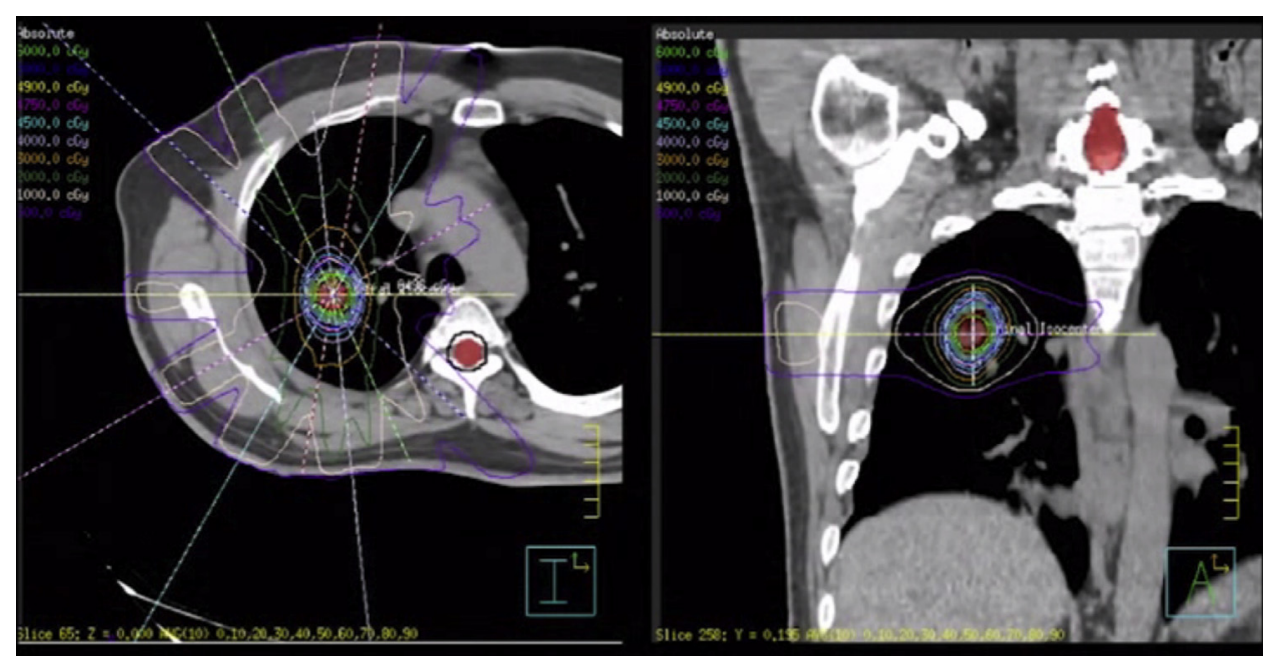

VIDEO 1. In this video, we share our experience with performing a robotic lobectomy for failed local control after stereotactic radiation to the right upper lobe. This patient was a 59-year-old man with squamous cell carcinoma of the right upper lobe, with adequate pulmonary function to undergo operative resection. The patient opted for stereotactic radiation, which included 50 Gy in 4 sessions. Local recurrence developed 10 months later. Robotic resection was performed safely and carried out as shown in this video. Video available at: http://www.jtcvsonline.org/article/S0022-5223(17)30703-1/addons. 
TABLE 6. Initial, preoperative, and pathologic staging for patients from MD Anderson Cancer Center cohort

\begin{tabular}{|c|c|c|c|c|c|c|c|}
\hline Age, $y$ & Sex & $\begin{array}{c}\text { Primary } \\
\text { malignancy }\end{array}$ & Histology & $\begin{array}{c}\text { Pre-SBRT } \\
\text { clinical stage }\end{array}$ & $\begin{array}{l}\text { Preoperative } \\
\text { clinical stage }\end{array}$ & Procedure & $\begin{array}{c}\text { Pathologic } \\
\text { stage }\end{array}$ \\
\hline 81 & M & NSCLC & Squamous & T2N0M0 & T2NOM0 & LUL lobectomy & T3N0M0 \\
\hline 72 & M & NSCLC & Squamous & T1N0M0 & T1N0M0 & RLL lobectomy & T1N0M0 \\
\hline 81 & $\mathrm{~F}$ & NSCLC & Squamous & T1N0M0 & T1N0M0 & $\begin{array}{l}\text { VATS RUL lobectomy } \\
\text { (extrapleural) }\end{array}$ & T2NOM0 \\
\hline 75 & M & NSCLC & Adenocarcinoma & T1N0M0 & T2NOMO & RML lobectomy & T2N1M0 \\
\hline 77 & M & NSCLC & Adenocarcinoma & T1N2M0 & T2NOM0 & RUL lobectomy (extrapleural) & T2NOM0 \\
\hline 74 & $\mathrm{~F}$ & NSCLC & Squamous & T1N0M0 & T2NOMO & RUL wedge (extrapleural) & T2NOM0 \\
\hline 73 & $\mathrm{~F}$ & NSCLC & Squamous & T2NOMO & T2NOM0 & LLL basilar segmentectomy & T2NOM0 \\
\hline 72 & $\mathrm{~F}$ & NSCLC & Atypical carcinoid & T4N0M0 & T4N0M0 & RLL wedge & T4N0M1 \\
\hline 81 & $\mathrm{~F}$ & NSCLC & Squamous & T2NOM0 & T2NOM0 & LLL lobectomy, pericardial fat flap & T2N1M0 \\
\hline 73 & M & NSCLC & Squamous & T1N0M0 & T1N0M0 & $\begin{array}{l}\text { Bilobectomy, subsequent } \\
\text { completion pneumonectomy, PA } \\
\text { plasty }\end{array}$ & T2NOM0 \\
\hline 69 & $\mathrm{~F}$ & NSCLC & Squamous & T1N0M0 & T2NOMO & LUL lobectomy, ICM flap & T3N0M0 \\
\hline 72 & M & NSCLC & Squamous & T1NOM0 & T2NOM0 & LLL lobectomy, ICM flap & T2N0M0 \\
\hline 59 & M & NSCLC & Squamous & T1N0M0 & T2NOM0 & RATS RUL lobectomy & T2N0M0 \\
\hline 69 & M & NSCLC & Adenocarcinoma & T1N0M0 & T1N0M0 & LUL lobectomy & $\mathrm{T} 2 \mathrm{~N} 2 \mathrm{M} 0$ \\
\hline 89 & $\mathrm{~F}$ & NSCLC & Adenocarcinoma & T1N0M0 & T2NOM0 & LLL wedge & T3N0M0 \\
\hline 53 & M & Esophageal & Adenocarcinoma & IV & & LLL superior segmentectomy & \\
\hline 45 & M & Colorectal & Adenocarcinoma & IV & & LUL trisegmentectomy & \\
\hline 74 & $\mathrm{~F}$ & Colorectal & Adenocarcinoma & IV & & LLL lobectomy, ICM flap & \\
\hline 46 & M & Colorectal & Adenocarcinoma & IV & & RLL wedge, diaphragm resection & \\
\hline 67 & $\mathrm{~F}$ & Colorectal & Adenocarcinoma & IV & & $\begin{array}{l}\text { RLL, intrapericardial, resection } \\
\text { LA, serratus anterior flap }\end{array}$ & \\
\hline
\end{tabular}

$S B R T$, Stereotactic body radiotherapy; $N S C L C$, non-small cell lung cancer; $L U L$, left upper lobe; $R L L$, right lower lobe; VATS, video-assisted thoracic surgery; $R U L$, right upper lobe; $R M L$, right middle lobe; $L L L$, left lower lobe; $P A$, pulmonary artery; $I C M$, intercostal muscle; $R A T S$, robotic-assisted thoracoscopic surgery; $L A$, left atrium.

with inherent limitations of current imaging strategies and relatively short-term follow-up. ${ }^{20-23}$ We have found a combination of CT scanning, PET imaging, and transthoracic needle biopsies to be helpful in guiding our follow-up of these patients. It is clear that future investigations will be imperative to identify the best way of surveilling such patients after stereotactic radiation. Although the exact rate of local recurrence remains unknown and merits separate investigation, it is clear that once recurrence has been identified, the optimal management of local recurrence after SBRT has not been well defined.

In recent years, there continues to be an increasing level of evidence suggesting that SBRT may offer operable patients with early-stage disease potentially comparable rates of local recurrence, disease-specific survival, and potential cost advantage compared with surgical resection. ${ }^{24-26}$ In addition, SBRT has become an attractive option for patients with small pulmonary metastases from other primary malignancies, yet the outcomes are not yet known. As the scope of SBRT continues to expand beyond inoperable patients, the feasibility, safety, and outcomes of salvage resections must be carefully considered.

In this study, we describe the largest series of pulmonary resection after local SBRT failure reported to date, along with a cumulative review that incorporates all patients who have been previously reported. At MDACC, we operated on 21 patients, among whom comorbidities were not insignificant. In a large review of the Society of Thoracic Surgeons (STS) General Thoracic (GT) database, patients undergoing anatomic lung resection between 2000 and 2010 had a mean age of 67.1 years, a diabetes frequency of $16 \%$, and a previous smoking rate of $73 \% .{ }^{27}$ In this same group of patients, the mean FEV1 was $80.8 \%$ of predicted, and the mean DLCO was $73.2 \%$ of predicted. Only $9.9 \%$ of these patients underwent preoperative radiation therapy. Of note, patients in our cohort had a median age of 73 years, were all irradiated, and demonstrated higher frequencies of diabetes, chronic obstructive pulmonary disease, and previous smoking. Median preoperative FEV1 and DLCO were $69 \%$ and $57 \%$, respectively. Further, the patients in this series were considered borderline operative candidates before radiation and were likely even less robust at the time of surgical resection, as suggested by the diminution in DLCO after SBRT. Therefore, it is not surprising that the incidence of pulmonary complications was higher $(33.3 \%)$ than the $20.3 \%$ reported in the study by Ceppa and colleagues. ${ }^{27}$ In addition, Kozower and colleagues, ${ }^{28}$ who also used the STS-GT database, examined patients who underwent pulmonary resection for lung cancer between 2002 and 2008, aiming to develop risk models for predicting major postoperative morbidity and mortality. In 
TABLE 7. Postoperative course, MD Anderson Cancer Center group

\begin{tabular}{llcc}
\hline & $\mathbf{N}(\%)$ & Mean (range) & $\mathbf{9 5} \% \mathbf{C I}$ \\
\hline ICU postoperative & $2(9.5)$ & & $1.17-30.3$ \\
Initial ICU stay, d & & $5.5(2-9)$ & -39.0 to 50.0 \\
Readmission to ICU & $2(9.5)$ & & $1.17-30.3$ \\
Prolonged postoperative vent & $1(4.8)$ & & $0.12-23.8$ \\
Pulmonary complications & $7(33.3)$ & $14.6-57.0$ \\
$\quad$ Pneumonia & $1(4.8)$ & $0.12-23.8$ \\
$\quad$ Respiratory arrest & $1(4.8)$ & $0.12-23.8$ \\
$\quad$ ARDS & $1(4.8)$ & $0.12-23.8$ \\
$\quad 2$ Discharged with tube & $2(9.5)$ & $1.17-30.3$ \\
Atrial arrhythmia & $3(14.3)$ & $3.04-36.3$ \\
Diarrhea & $1(4.8)$ & & $0.12-23.8$ \\
Bleeding requiring transfusion & $1(4.8)$ & & $0.12-23.8$ \\
Urinary tract infection & $1(4.8)$ & & $0.12-23.8$ \\
Thrombophlebitis & $1(4.8)$ & & $0.12-23.8$ \\
Retinal detachment & $1(4.8)$ & & $0.12-23.8$ \\
Death & $1(4.8)$ & & $0.12-23.8$ \\
Any complication & $6(28.6)$ & & $11.3-52.2$ \\
Length of stay (d) & & $7.1(2-23)$ & $4.53-9.66$ \\
Chest tube duration (d) & & $4.8(0.7-14)$ & $3.30-6.35$ \\
\hline CI, Confidence interval; $I C U$, intensive care unit; $A R D S$, acute respiratory distress \\
syndrome.
\end{tabular}

this study, overall mortality was $2.2 \%$, and FEV1 was a significant predictor of perioperative mortality. Although our mortality is suboptimally high at $1(4.8 \%)$, it is important to recognize the limitations of our small sample size and the considerably higher-risk population in whom we operated. An important consideration, when comparing our data with that of the STS-GT database, is the fact that only $8.1 \%$ of these patients underwent minimally invasive procedures, which is less than in the general population captured in the database and may be a contributing factor in the rate of postoperative complications. Although the majority of the patients in our series were managed by thoracotomy, it is not clear whether more could have feasibly

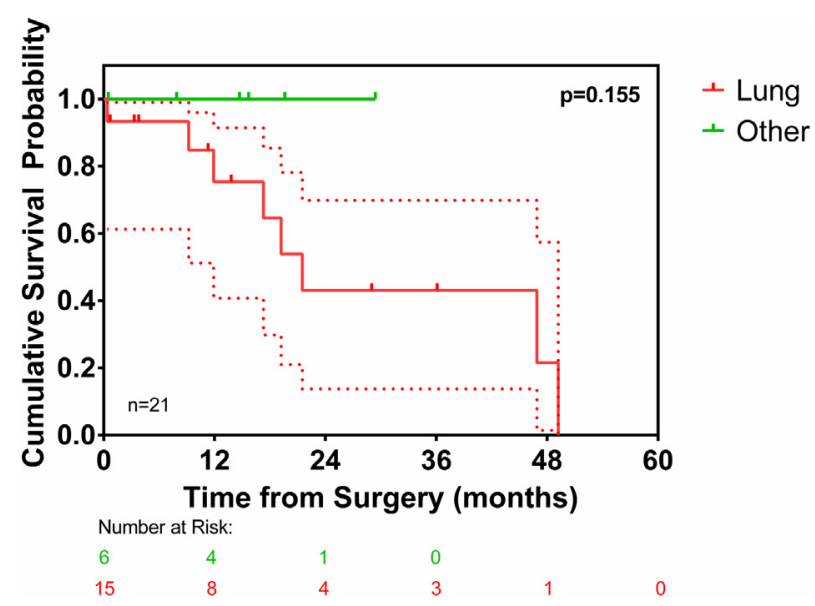

FIGURE 2. Survival from the time of surgery for the MDACC group, $\mathrm{n}=21$; solid red line represents NSCLC, solid green line represents metastatic disease, and dotted lines represent $95 \%$ confidence intervals.

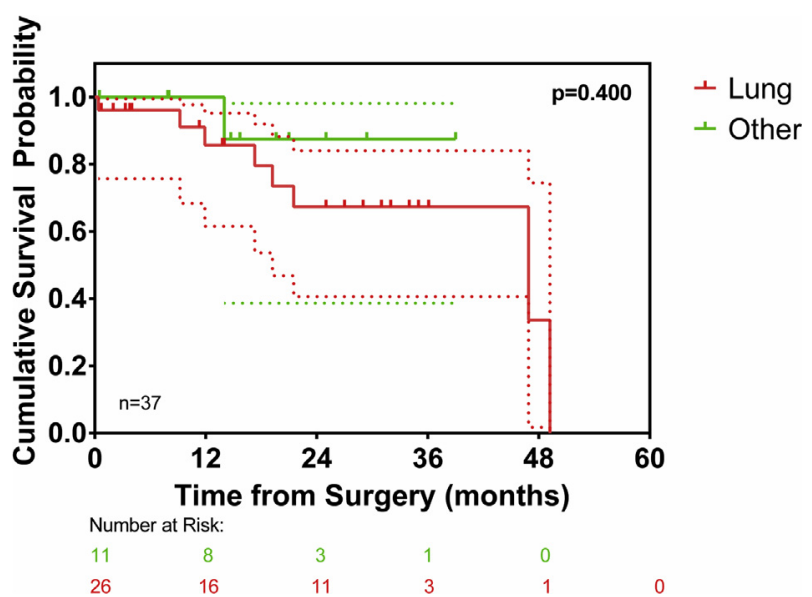

FIGURE 3. Survival from surgery for cumulative group, $\mathrm{n}=37$; solid red line represents NSCLC, solid green line represents metastatic disease, and dotted lines represent $95 \%$ confidence intervals.

been managed with less-invasive techniques, and this will be an important area to further investigate.

\section{Study Limitations}

This study is limited by both its size and the opportunity for selection bias. Although our numbers are small, this currently represents the largest series of its type, thus prompting us to combine our cohort with patients previously reported in the medical literature. As a case series, the level of evidence is limited. We do recognize that the patients who underwent salvage resection probably do not represent the full spectrum of individuals who fail SBRT and that selection bias most likely influenced who did or did not receive salvage resection. We acknowledge that those patients offered surgical resection were carefully selected, and we cannot know how they compare in terms of functional status and comorbidities with individuals who failed SBRT and were not offered salvage resection. In addition, there were elements of missing data for some of the patients; however, we believe that such missing variables were random rather than systematic and did not inherently bias the outcomes.

\section{CONCLUSIONS}

Although there are significant limitations to the study, our findings demonstrate that resection after local failure of SBRT in highly select individuals is feasible and safe, and has an overall acceptable morbidity and mortality, albeit higher than what is typically observed in nonirradiated patients. In considering salvage resection, we recommend careful consideration of the patient's performance status and the likely extent of required resection, to be discussed thoughtfully both with the patient and in a multidisciplinary tumor board setting. Further studies clearly are warranted to establish treatment algorithms for those patients who demonstrate locally persistent or recurrent disease after 
SBRT and to clinically characterize the most appropriate operative candidates.

\section{Webcast}

You can watch a Webcast of this AATS meeting presentation by going to: http://webcast.aats.org/2016/Video/ Tuesday/05-17-16_Ballroom_IV_1630_Antonoff-800.mp4.

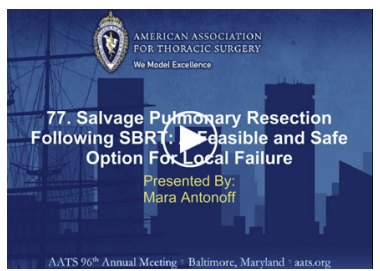

\section{Conflict of Interest Statement}

Authors have nothing to disclose with regard to commercial support.

\section{References}

1. Ginsberg RJ, Rubinstein LV. Randomized trial of lobectomy versus limited resection for T1 N0 non-small cell lung cancer. Lung Cancer Study Group. Ann Thorac Surg. 1995;60:615-23.

2. Roesch J, Andratschke N, Guckenberger M. SBRT in operable early stage lung cancer patients. Transl Lung Cancer Res. 2014;3:212-24.

3. Vansteenkiste J, De Ruysscher D, Eberhardt WE, Lim E, Senan S, Felip E, et al Early and locally advanced non-small-cell lung cancer (NSCLC): ESMO Clinical Practice Guidelines for diagnosis, treatment and follow-up. Ann Oncol. 2013;24(Suppl 6):vi89-i98.

4. Timmerman RD, Kavanagh BD, Cho LC, Papiez L, Xing L. Stereotactic body radiation therapy in multiple organ sites. J Clin Oncol. 2007;25:947-52.

5. Timmerman R, McGarry R, Yiannoutsos C, Papiez L, Tudor K, DeLuca J, et al. Excessive toxicity when treating central tumors in a phase II study of stereotactic body radiation therapy for medically inoperable early-stage lung cancer. J Clin Oncol. 2006;24:4833-9.

6. Lagerwaard FJ, Haasbeek CJ, Smit EF, Slotman BJ, Senan S. Outcomes of riskadapted fractionated stereotactic radiotherapy for stage I non-small-cell lung cancer. Int J Radiat Oncol Biol Phys. 2008;70:685-92.

7. Timmerman R, Paulus R, Galvin J, Michalski J, Straube W, Bradley J, et al. Stereotactic body radiation therapy for inoperable early stage lung cancer. JAMA. 2010;303:1070-6.

8. Baumann P, Nyman J, Hoyer M, Wennberg B, Gagliardi G, Lax I, et al. Outcome in a prospective phase II trial of medically inoperable stage I non-small-cell lung cancer patients treated with stereotactic body radiotherapy. J Clin Oncol. 2009; 27:3290-6.

9. Palma D, Visser O, Lagerwaard FJ, Belderbos J, Slotman BJ, Senan S. Impact of introducing stereotactic lung radiotherapy for elderly patients with stage I nonsmall-cell lung cancer: a population-based time-trend analysis. J Clin Oncol. 2010;28:5153-9.

10. Crabtree T, Puri V, Timmerman R, Fernando H, Bradley J, Decker PA, et al. Treatment of stage I lung cancer in high-risk and inoperable patients: comparison of prospective clinical trials using stereotactic body radiotherapy (RTOG 0236), sublobar resection (ACOSOG Z4032), and radiofrequency ablation (ACOSOG Z4033). J Thorac Cardiovasc Surg. 2013;145:692-9.

11. Timmerman RD, Fernando HC. A radiation oncologist's and thoracic surgeon's view on the role of stereotactic ablative radiotherapy for operable lung cancer. Semin Thorac Cardiovasc Surg. 2013;25:8-13.

12. Chang JY, Senan S, Paul MA, Mehran RJ, Louie AV, Balter P, et al. Stereotactic ablative radiotherapy versus lobectomy for operable stage I non-small-cell lung cancer: a pooled analysis of two randomised trials. Lancet Oncol. 2015;16:630-7.

13. Rosen JE, Salazar MC, Wang Z, Yu JB, Decker RH, Kim AW, et al. Lobectomy versus stereotactic body radiotherapy in healthy patients with stage I lung cancer. J Thorac Cardiovasc Surg. 2016;152:44-54.e49.

14. Allibhai Z, Cho BCJ, Taremi M, Atallah S, Hope A, Hwang D, et al. Surgical salvage following stereotactic body radiotherapy for early-stage NSCLC. Eur Respir J. 2012;39:1039-42.

15. Chen F, Matsuo Y, Yoshizawa A, Sato T, Sakai H, Bando T, et al. Salvage lung resection for non-small cell lung cancer after stereotactic body radiotherapy in initially operable patients. J Thorac Oncol. 2010;5:1999-2002.

16. Neri S, Takahashi Y, Terashi T, Hamakawa H, Tomii K, Katakami N, et al. Surgical treatment of local recurrence after stereotactic body radiotherapy for primary and metastatic lung cancers. J Thorac Oncol. 2010;5:2003-7.

17. Taira N, Kawabata T, Ichi T, Kushi K, Yohena T, Kawasaki H, et al. Salvage oper ation for late recurrence after stereotactic body radiotherapy for lung cancer: two patients with no viable cancer cells. Ann Thorac Surg. 2014;97:2167-71.

18. Crabtree TD, Puri V, Robinson C, Bradley J, Broderick S, Patterson GA, et al. Analysis of first recurrence and survival in patients with stage I non-small cell lung cancer treated with surgical resection or stereotactic radiation therapy. $J$ Thorac Cardiovasc Surg. 2014;147:1183-92.

19. Lindberg K, Nyman J, Riesenfeld Kallskog V, Hoyer M, Lund JÅ, Lax I, et al. Long-term results of a prospective phase II trial of medically inoperable stage I NSCLC treated with SBRT - the Nordic experience. Acta Oncol. 2015;54: 1096-104.

20. Halpenny D, Ridge CA, Hayes S, Zheng J, Moskowitz CS, Rimner A, et al. Computed tomographic features predictive of local recurrence in patients with early stage lung cancer treated with stereotactic body radiation therapy. Clin Imaging. 2015;39:254-8.

21. Dahele M, Palma D, Lagerwaard F, Slotman B, Senan S. Radiological changes after stereotactic radiotherapy for stage I lung cancer. J Thorac Oncol. 2011;6:1221-8.

22. Dahele M, Pearson S, Purdie T, Bissonnette JP, Franks K, Brade A, et al. Practical considerations arising from the implementation of lung stereotactic body radiation therapy (SBRT) at a comprehensive cancer center. J Thorac Oncol. 2008;3:1332-41.

23. Hoopes DJ, Tann M, Fletcher JW, Forquer JA, Lin PF, Lo SS, et al. FDG-PET and stereotactic body radiotherapy (SBRT) for stage I non-small-cell lung cancer. Lung Cancer. 2007;56:229-34.

24. Louie AV, Rodrigues G, Hannouf M, Zaric GS, Palma DA, Cao JQ, et al. Stereotactic body radiotherapy versus surgery for medically operable stage I non-smallcell lung cancer: a Markov model-based decision analysis. Int J Radiat Oncol Biol Phys. 2011;81:964-73.

25. Crabtree TD, Denlinger CE, Meyers BF, El Naqa I, Zoole J, Krupnick AS, et al. Stereotactic body radiation therapy versus surgical resection for stage I non-small cell lung cancer. J Thorac Cardiovasc Surg. 2010;140:377-86.

26. Puri V, Crabtree TD, Kymes S, Gregory M, Bell J, Bradley JD, et al. A comparison of surgical intervention and stereotactic body radiation therapy for stage I lung cancer in high-risk patients: a decision analysis. J Thorac Cardiovasc Surg. 2012;143:428-36.

27. Ceppa DP, Kosinski AS, Berry MF, Tong BC, Harpole DH, Mitchell JD, et al. Thoracoscopic lobectomy has increasing benefit in patients with poor pulmonary function: a Society of Thoracic Surgeons Database analysis. Ann Surg. 2012;256: 487-93.

28. Kozower BD, Sheng S, O’Brien SM, Liptay MJ, Lau CL, Jones DR, et al. STS database risk models: predictors of mortality and major morbidity for lung cancer resection. Ann Thorac Surg. 2010;90:875-83.

Key Words: lung cancer surgery, radiation therapy

\section{Discussion}

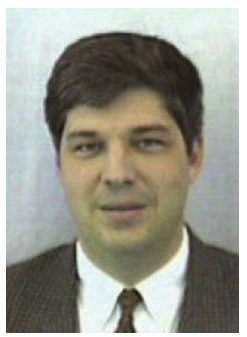

Dr Stephen R. Hazelrigg (Springfield, Ill). Your article is well written and obviously timely, as you mentioned in the conclusion, because of the increased use of SBRT both in nonoperative patients and seemingly in operative patients. I am unaware of any literature that would support it in operative patients with early-stage lung cancer that would say it is even equivalent, although I know that the local recurrence rates have been decreasing. 
When I looked at your results, they were very good. These patients did well. It was a highly selected group, as you noted. It may not represent every patient who would show up in our office with a recurrence after SBRT, but it looked to me that the way we would go about evaluating these patients if we were considering a salvage operation is no different than the way we would evaluate patients who just came in our office with a lung cancer who had not had previous radiation. I understand they had a few more adhesions, but I was unable to really come up with anything else that would change the way I would evaluate one of these patients when they came to our office. So my first question is, does that seem accurate, or based on what you have seen here, is there something you do different in this group of patients?

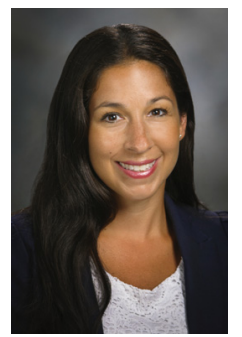

Dr Antonoff. In terms of evaluating these patients preoperatively, certainly once they have already failed locally, it is important to restage them, which would be very similar to the way that you would evaluate any other new patient, as you suggested. In terms of going through the process of getting another PET scan, brain magnetic resonance imaging, or restaging the mediastinum, we are careful about that. It is safe to say that in many patients with early-stage disease, there are situations in which we don't all necessarily pathologically stage the mediastinum. We may rely heavily on imaging strategies. But in patients who have already had a recurrence, we may be more aggressive in terms of pathologic staging of the mediastinum with an endobronchial ultrasound, but otherwise I would agree that the way we would evaluate these patients is similar to any other patient with early-stage disease.

Dr Hazelrigg. You ended up performing a lobectomy in three fourths of these, or 1 had a bilobectomy, so obviously those patients would have been surgical candidates 16 months before. Did any of these patients see thoracic surgeons before opting for the SBRT? I'm sure some were perfectly legitimate, but the question is, were they seen and screened and determined that they were not surgical candidates?

Dr Antonoff. The practice at our institution is for patients generally to be seen by radiation oncology, thoracic medical oncology, and thoracic surgery when there is a question of which modality (or modalities) will be involved in the treatment plan. All of these patients in our series underwent operation at our institution, but they did not all receive their SBRT at our institution, and I cannot speak to the types of evaluation that occur elsewhere. Also, to clarify, you stated that, due to the extent of resection, those patients who underwent at least a lobectomy would have tolerated a resection at initial presentation. It is important to note that a few patients in this cohort were directed toward SBRT not because of irreversible comorbidities, such as poor pulmonary reserve, but rather due to temporary issues; for example, 1 patient had a concurrent pancreatic cancer that required a Whipple procedure.

Dr Hazelrigg. With regard to the adhesions that you saw when you reoperated on these patients, we have had experience with only 2 and have seen few adhesions in those. Do you think it has to do with where the tumor is? I mean, if it is out on the periphery, do you get more adhesions than not? Do you have any insight into that?

Dr Antonoff. I don't have specific insight into that. We did look at all of these patients in terms of where the tumor was relative to the edge of the pleura and size, and there did not seem to be any specific patterns in predicting who might have had significant adhesions, although I will say for the patients who received SBRT at our institution, generally there are pretty uniform standards as to who may receive SBRT. So it is not usually central lesions, near the vessels, or anything like that in terms who might receive this treatment.

Dr Hazelrigg. I suppose my last statement is really just a comment. I hope your good results with regard to salvaging these patients does not result in the thought, "Oh, we may as well give them SBRT first because we can always salvage them with surgery at the end."

Dr Antonoff. I appreciate your comment and I actually would love the opportunity to reply to it. I agree that that your point is critical and timely, and is such concerns certainly impacted some of the reluctance that we had in conducting this study and writing the paper. I will tell you that, again, this is a really very highly-selected group of patients, and during the same time period of this study, 1400 patients received SBRT at our institution; about 1300 for primary lung cancer and about 100 for metastatic disease. Our radiation oncologists at our institution, who don't have a similar database to the one that we maintain in our surgical department, tell us that they are aware of 38 patients who had recurrence, which would be approximately a $2.7 \%$ local recurrence rate. We do not have any way to validate that information. It is hard to know if patients have recurrence and they don't come back or let anybody know. It is challenging to measure the validity of that estimate. These patients may be difficult to follow, not only because of the lack of guidelines regarding proper surveillance but also because of the characteristics of the cohort. They tend to be high-risk patients, patients with lots of comordibities, patients who may not be able to travel easily for follow-up. Our colleagues in radiation oncology don't have an easy time tracking these individuals, but it's important that we all work together to solve these issues, and to 
track outcomes for patients with the disease which we treat. So I agree that our patients had acceptable outcomes given the particularly high-risk nature of the group-33\% with pulmonary complications; 1 death, which made it $5 \%$ mortality-but we really don't know what it would look like if we operated on all of the other potential recurrences: those which were known recurrences but not thought to be candidates for salvage, as well as those that may have recurred without our knowledge.

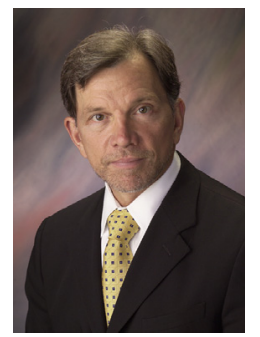

Dr James Luketich (Pittsburgh, Pa). Dr Hazelrigg pointed out some important considerations, namely, the central lesions are largely unknown. We are the principal investigator site for an Accuray trial looking at central lesions because of the concern that this treatment will melt away a bronchus. If you get near the bronchus intermedius, you will have problems. We know that much from the trial, but it is not yet at a point where we can report it, but there are significant problems with central lesions.

In terms of the peripheral lesions, your local recurrence rate, and, as you pointed out, this is not an accurate description of the local recurrence rate, is far in excess of a bad wedge. We are way over $30 \%$ to $40 \%$, and we are a thoracic group who perform the SBRT ourselves, so we are quick to point out the limitations. I think that is my main comment.

Are the thoracic surgeons involved in the decision making on who receives SBRT, and are you actually doing the conformal treatment and treatment planning?

Dr Antonoff. Thank you for sharing with us your insight from your experience. We are most often involved in the decision-making process to proceed with SBRT, although we are not usually involved in the treatment planning. We are very active in the follow-up and surveillance of patients whom we've seen, particularly if they may be surgical candidates.

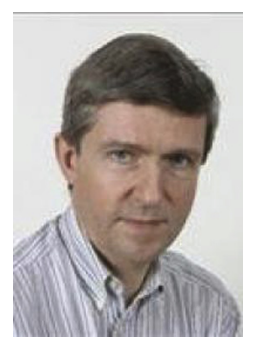

Dr Paul E. Van Schil (Antwerp, Belgium). Thank you for this important contribution. I think you should also present it in front of radiation oncologists. It is important that they are aware of it.

I have 2 short questions. Regarding the extent of resection, you had patients in whom you had to perform a bilobectomy or even a pneumonectomy, which also happened to us. Did you go back to the initial CT scan before the SBRT to see whether those patients could have had a lesser resection than what you had to do later on?

Dr Antonoff. The patient who specifically received a bilobectomy and ultimately a pneumonectomy was a patient in whom a pulmonary artery patch reconstruction was required, and then the pulmonary artery thrombosed and the patient required a completion pneumonectomy later during the hospitalization. That patient also is the same one who was our single mortality. It's a bit of an unusual case. Unfortunately, we have a small series, so it is hard to gather that kind of information, but the numbers continue to increase with these types of patients at our institution, and we plan to continue with these types of analyses.

Dr Van Schil. Second, preoperative staging is not that easy, because invariably you have a lot of fibrosis and inflammation after SBRT that can last for months or years. How do you decide whether to operate or not on a patient?

Dr Antonoff. I agree that we don't know how to follow these people, and we don't know necessarily from the PET scan. We do require that there is some sort of tissue evidence of persistent malignancy. In terms of the nodal stations, even with pathologic staging preoperatively, we were not correct $100 \%$ of the time. In $20 \%$ of the patients in whom we operated on, there actually was nodal disease at the time of surgery.

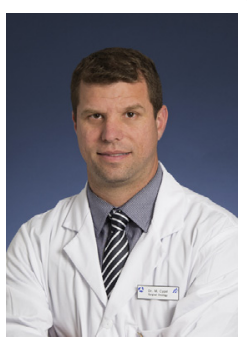

Dr Marcelo Cypel (Toronto, Ontario, Canada). Just to follow up on Dr Van Schil's question, in your resected specimens, was there cancer in all of them?

Dr Antonoff. Yes, absolutely. All of our patients had pathologic confirmation of malignancy prior to salvage surgery. That is why when we did the cumulative analysis, we chose not to include the single article that included 2 resections with no residual disease.

Dr Cypel. Did you have biopsies confirming the recurrence on all of them?

\section{Dr Antonoff. Yes, absolutely.}

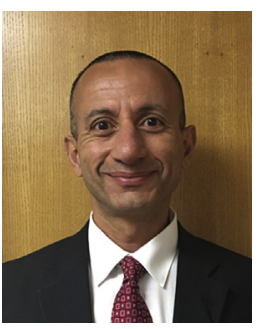

Dr Abbas E. Abbas (Philadelphia, $\mathrm{Pa}$ ). How was the determination of failure of SBRT made? Was it made by the radiation oncologists?

Dr Antonoff. In our series, it was a combination of determinations by our surgeons and radiation oncologists. If some of the surgeons in our group, when we see patients before therapy, do go the route of SBRT, many of us follow the patients and see them when they come back, and if it doesn't look as though they are responding as they should on their PET scans, we will get a biopsy and find out if there is residual disease.

Dr Abbas. What is the timeline for that? When do you trust the PET scan? 
Dr Antonoff. That is a challenge. We don't have a standard set of guidelines and that is part of the problem. Practices will vary, from getting a PET 4 to 6 months later, but we need to evaluate the best means of surveilling these patients.

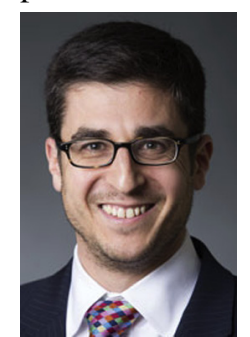

Dr David J. Finley (Lebanon, NH). I have 2 quick questions. For the specific stage after resection, you said that $20 \%$ of the patients were upstaged. Did they have nodal disease?

Dr Antonoff. That is correct.

Dr Finley. Was that N1 or N2 disease?

Dr Antonoff. Two of the patients had N1 disease, and 1 patient had N2.

Dr Finley. I saw on the graph that the patients who had high preoperative or pre-SBRT DLCOs $(100 \%$ and $80 \%$ ) had the most significant decrease in their DLCOs compared with the patients who had pre-SBRT DLCOs of $50 \%$ or $60 \%$. Is that something anyone has looked at across the 1100 SBRT cases you have at MDACC?

Dr Antonoff. We have not evaluated that, but it is certainly something that we would like to do.

Dr Finley. Because if we are talking about people who are operative and then we are going to cause their DLCO to decrease drastically, that is something to think about.

Dr Antonoff. Absolutely.

Dr Finley. You said that the majority of the patients underwent thoracotomy when they did this. Was this because of the concern that there were going to be more adhesions, and now that you have done them, do you think that you could do more of these minimally invasively?

Dr Antonoff. Yes, I think it was concern and surgeon preference, and as we accumulate more experience with these, some of them will be done open and some of them will be done minimally invasively. 